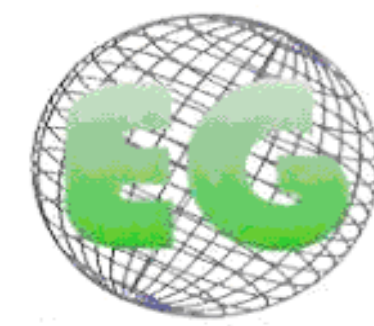

ISSN 1695-6141

$N^{\circ} 23$
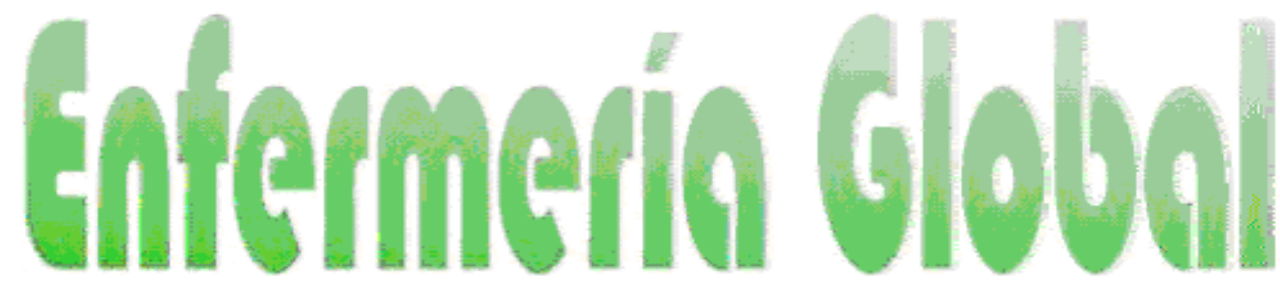

Revista electrónica trimestral de Enfermería

www.um.es/egloball

DOCENCIA - INVESTIGACIÓN

\title{
Intervención educativa para promover el conocimiento del trastorno de déficit de atención con hiperactividad (TDAH) con padres de familia de una institución educativa
}

Educational intervention to promote knowledge about attention deficit disorder with hyperactivity (ADHD) with parents of an educational institution

\author{
*Piedrahita S., LE., "*Henao Cardona, A., **Burgos, PA. \\ *Profesora Asociada. **Estudiante de Enfermería. Escuela de Enfermería. Facultad de Salud. Universidad del \\ Valle. Santiago de Cali. Colombia.
}

Palabras clave: Intervención educativa; Niños; TDAH; Padres.

Keywords: Educational intervention; Child; ADHD; Parents.

\section{RESUMEN}

Se realizó un proyecto de intervención educativa con el objetivo de promover el conocimiento del trastorno de déficit de atención con hiperactividad (TDAH) con padres de familia en una Institución Educativa de la ciudad de Cali, en el periodo enero-diciembre de 2009. La muestra estuvo conformada por 37 padres. Se diseñó la intervención en cuatro etapas: una inicial para identificar el nivel de conocimientos de los padres sobre el TDAH; la segunda etapa, en la cual se elaboró el programa educativo "Estrategias para identificación y manejo del escolar con TDAH"; en la tercera etapa se implementó el programa, y en la cuarta etapa se evaluó el impacto de las actividades educativas.

Los hallazgos antes de la intervención evidenciaron el desconocimiento de los padres respecto al TDAH. A partir de los resultados obtenidos después de la intervención educativa, se evidenció un aumento de bases teóricas y herramientas prácticas de los padres, con respecto a los conocimientos previos sobre el trastorno. La alta prevalencia del trastorno y el desconocimiento de los padres conllevan a la falta de remisión de los escolares para tratamiento o intervención.

Se llegó a la conclusión de que la intervención educativa muestra una significativa efectividad al elevar el nivel de conocimientos de los padres respecto al TDAH, su manejo y remisión. La ganancia de conocimientos aporta mayor información y posibilita un diagnóstico y tratamiento oportuno, lo cual conlleva a la disminución del deterioro tanto escolar como psicosocial en los menores. 


\section{ABSTRACT}

An educational intervention project was performed with the aim of promoting knowledge about attention deficit disorder with hyperactivity (ADHD), with parents in an Educational Institution of Cali city in the period January to December 2009. The sample consisted of 37 parents. The intervention was designed in four stages: an initial one to identify the level of the parents' knowledge about ADHD. During the the second stage, the program "Strategies to identification and management of schoolboys and schoolgirls with ADHD" was developed; in the third stage the program was implemented, and in the fourth stage the impact of educational activities was evaluated .The findings before intervention showed the parents' unawareness about ADHD. From the post-educational intervention results obtained, an increase in the theoretical base and practical tools of parents was shown with regard to preliminary knowledge about disorder. The high prevalence of disorder and the parents' unawareness leads to the absence of schoolchildren's remission for treatment or intervention. The conclusion was that educational intervention shows a significant effectiveness because it increases the level of knowledge of the parents about ADHD, its management and remission. The knowledge gain brings more information, facilitates a diagnostic and opportune treatment. Finally it leads to a decrease in school and psychological deterioration in childhood.

\section{INTRODUCCIÓN}

El trastorno por déficit de atención/hiperactividad (TDAH) es un trastorno del desarrollo neurológico infantil frecuente que se caracteriza por la presencia persistente de inatención, hiperactividad e impulsividad ${ }^{(1)}$. Es el problema de comportamiento más común durante la infancia; constituye la enfermedad crónica más frecuente del periodo escolar y representa un problema complejo porque aparece en edades tempranas y repercute en la vida del niño y su grupo familiar. Se ha mencionado la influencia del TDAH en el comportamiento violento del joven y el mayor riesgo de deserción escolar y de drogadicción. Y adicionalmente, existe la probabilidad de que el trastorno persista a lo largo de la vida. ${ }^{(2)}$

El diagnóstico del TDAH se realiza generalmente mediante la aplicación del Manual de Clasificación y Diagnóstico de las Enfermedades Mentales (DSMIV-TR) de la Asociación Americana de Psiquiatría ${ }^{(2)}$, el cual requiere la presencia de un cierto número de síntomas que se deben presentar más frecuentemente y de una forma más intensa que en los niños que no presentan el trastorno, de la misma edad y nivel de desarrollo. Deben presentarse antes de los 7 años, en más de un lugar (casa, colegio, etc.), durante un período superior a los 6 meses y deben interferir con las actividades sociales y académicas del niño, o con las actividades ocupacionales del adolescente o del adulto (1). Así mismo, la clasificación internacional de enfermedades (CIE 10) lo contempla dentro de la categoría del trastorno del comportamiento y de las emociones que comienzan habitualmente en la infancia $y$ adolescencia. $^{(3)}$

Su etiología probablemente sea multifactorial y posiblemente los factores genéticos tengan una gran relevancia interactuando con los procesos orgánicos y ambientales ${ }^{(4)}$. El TDAH actualmente representa el primer motivo de consulta en los servicios de salud mental de niños ${ }^{(1)}$, generalmente se diagnostica en los primeros años de la enseñanza primaria. No obstante algunos síntomas están ya presentes antes de los 7 años de edad y con frecuencia persiste en la adolescencia y en la vida adulta.

La prevalencia informada por el DSM-IV está en el 3-5\% de la población escolar (1) (5). Los estudios realizados recientemente en Colombia informan de una prevalencia mucho mayor, que afecta al $16 \%$ de esta población ${ }^{(1)}$. Sólo el $7.4 \%$ recibe un diagnóstico de confirmación mediante una entrevista psiquiátrica estructurada y solo el $6.6 \%$ de los afectados recibe 
tratamiento ${ }^{(1)}$ lo cual tiene importancia en el pronóstico, ya que se ha reportado el mayor riesgo de deserción escolar, de drogadicción, la mayor probabilidad de infracciones a la ley y se ha asociado con el uso de sustancias psicoactivas en el adolescente. ${ }^{(2)}$

El $87 \%$ de los niños presentan un diagnóstico comórbido y el $67 \%$ por lo menos dos asociados, siendo los más frecuentes el trastorno de conducta de oposición desafiante (TOD) y el trastorno de desarrollo de la coordinación (TDC) $)^{(6)}$, los cuales interfieren significativamente con el aprendizaje escolar o las actividades de la vida diaria. Adicionalmente se encuentran además asociados a trastornos de la comunicación, elevando el riesgo de presentar dificultades en el aprendizaje de la lectoescritura y trastornos del aprendizaje como dislexia, discalculia y disgrafía ${ }^{(7)}$. La depresión también muestra una fuerte asociación con el TDAH, diversos estudios coinciden en señalar que alrededor del $30 \%$ de los niños con TDAH tienen asociado algún trastorno afectivo (depresión mayor, trastorno bipolar o trastorno distímico). ${ }^{(6)}$

El TDAH es un trastorno aún poco reconocido, por lo que menos de la mitad de los pacientes afectados ha recibido un diagnóstico adecuado, y aún dentro de éstos, pocos reciben el adecuado tratamiento. Las bajísimas frecuencias de antecedentes de intervención terapéutica halladas en una muestra evaluada en Colombia ${ }^{(3)}$ sugiere que la falta de tratamiento se relaciona con el desconocimiento de la existencia del trastorno por parte de los padres, los maestros y los profesionales de la salud, con los problemas de accesibilidad a los servicios asistenciales y con los temores que todavía genera en los familiares de los niños la intervención farmacológica.

Es importante que los padres de familia tengan conocimientos frente a este trastorno, que les brinden la posibilidad de adquirir herramientas para su identificación y remisión para tratamiento profesional oportuno de los escolares. En el caso de los padres, suelen ser ellos los que valoran sí existe o no un problema, y quienes deciden si necesitan ayuda profesional $^{(8)}$ Ya que las probabilidades de identificar el problema dependen de la perturbación que el niño pueda crear en su ambiente familiar y en el medio escolar.

Algunos trabajos sobre el trastorno de déficit de atención con hiperactividad muestran especial interés en aspectos relacionados con las manifestaciones sintomáticas y las características neurológicas que presentan los niños; en los últimos años, se han desarrollado investigaciones sobre factores psicosociales y contextuales que afectan la evolución del trastorno, mostrando la importancia del papel de la familia y la escuela como eje fundamental en su manejo ${ }^{(8)}$ Teniendo en cuenta la alta frecuencia así como su influencia sobre el rendimiento escolar se propone un trabajo de investigación desde enfermería a través de la implementación de una estrategia de intervención educativa en salud que promueva el conocimiento del TDAH, en padres de familia. Se ha demostrado que mediante la educación y el conocimiento del trastorno se logra una actuación temprana y eficaz por parte de los padres. ${ }^{(9)}$

\section{MATERIAL Y MÉTODO}

Se realizó un proyecto de intervención educativa con padres de familia en una institución educativa del sector oficial de la ciudad de Cali. La población del estudio estuvo conformada por 37 padres de familia de escolares matriculados en el periodo enero-diciembre de 2009. Con edades entre los 5 y 10 años, que cursan de transición a quinto grado de primaria, se incluyeron estudiantes de sexo masculino y femenino. 
Inicialmente, se presentó el proyecto a las directivas de la Institución educativa para su revisión y aprobación. El estudio se diseñó en cuatro etapas: una inicial o diagnóstica que buscaba identificar el nivel de conocimientos de los padres sobre el trastorno de déficit de atención con hiperactividad (TDAH), para lo cual se diseñó un instrumento de valoración por parte de las autoras, constituido por dos partes: datos sociodemográficos y epidemiológicos y en la segunda parte, los conocimientos sobre el trastorno.

En la segunda etapa, se elaboró un programa educativo con el objetivo de incrementar el nivel de conocimientos, utilizando para su diseño las necesidades de aprendizaje identificadas en el instrumento inicial.

En la tercera etapa, de intervención, se procedió a la implementación del programa creado por las autoras denominado "Estrategias para identificación y manejo del escolar con TDAH", conformado por tres módulos con los siguientes ejes temáticos: Conocimiento del TDAH, frecuencia y etiología; manifestaciones clínicas y tratamiento; complicaciones, herramientas para manejo por parte de los padres, profesionales e instituciones para remisión y manejo interdisciplinario.

Y una cuarta etapa, que buscaba evaluar el impacto de las actividades educativas; para lo cual se aplicó la encuesta utilizada en la etapa diagnóstica o de identificación de necesidades. Este programa se impartió en el transcurso de cinco encuentros teóricoprácticos en grupo, que tuvieron lugar durante un mes con una duración aproximada de dos horas cada uno. En cada sesión se abordaron los temas como un proceso de construcción conjunta de conocimientos a partir de situaciones problema mediante dinámicas grupales como los grupos focales y el análisis de casos.

El procesamiento de los datos se efectuó con ayuda del paquete estadístico SPSS 11.0 tanto para la elaboración de la base de datos, la tabulación y análisis de los mismos. ${ }^{(10)}$

En cuanto a los aspectos éticos, se realizó una reunión con los padres de familia para explicarles los objetivos del estudio y, posteriormente, se procedió a diligenciar el formato de recolección de los datos, previa obtención del consentimiento informado. ${ }^{(11)}$

\section{RESULTADOS.}

Los resultados del estudio incluyen la descripción de las características sociodemográficas de la población y la evaluación del nivel de conocimientos antes y después de la intervención educativa.

\section{Datos sociodemográficos:}

De los 37 padres de familia con los que se realizó el estudio el $68 \%$ fueron madres, el $16 \%$ padres, $11 \%$ abuela y el $5 \%$ tío. Con relación a la edad, $16 \%$ tiene entre $20-25$ años, $26 \%$ entre 26-30 años, 16\% 31-35 años, 10\% 36-40 años, 21\% 41-45 años y el 11\% entre 46-50 años. Con respecto al género, $79 \%$ es femenino y el $21 \%$ restante masculino. En cuanto a la Escolaridad $37 \%$ realizó estudios de primaria, $42 \%$ secundaria, $11 \%$ tecnológico, 5\% Universitario y el 5\% no había realizado ningún estudio.

Con relación al estado civil, $21 \%$ eran solteros, $16 \%$ casados, $53 \%$ en unión libre y $10 \%$ divorciados. Con relación al número de hijos, el 5\% no tiene hijos, el $11 \%$ tiene un hijo, el $37 \%$ tiene 2 hijos, el $26 \%$ tiene tres hijos, el 16\% tiene cuatro hijos y el $5 \%$ tiene cinco hijos. 
Con relación al estrato socioeconómico, el $5 \%$ pertenece al estrato socioeconómico 1 , el $37 \%$ en estrato 2 y el $58 \%$ en estrato 3 . En lo relativo a la ocupación el $74 \%$ respondió que sí tiene ocupación, el 16\% que no tiene ocupación y el 10\% de los asistentes no respondieron.

\section{Nivel de conocimiento:}

Antes de la implementación del programa educativo, se identificó algún conocimiento con relación al TDAH así: el $42 \%$ de los participantes reconoció poseer algún conocimiento y un 58\% reconoció no poseer ningún conocimiento al respecto. Con relación a la frecuencia del trastorno, $27 \%$ de los padres lo consideraron como una alteración muy frecuente, 52\% como poco frecuente y $21 \%$ no sabe cuán frecuente es este trastorno. Con relación a las manifestaciones clínicas, un 48\% consideraba que el TDAH tenía como característica la agresividad; mientras que $52 \%$ no sabía. El $47 \%$ de los padres consideraba que los niños con TDAH presentan indisciplina y el $53 \%$ no sabía. $32 \%$ de los padres consideró que el niño con TDAH presenta bajo rendimiento académico, el $21 \%$ consideró que no y el $47 \%$ no sabía si se presenta o no. Lo cual expresaron como: "Por su comportamiento, le presta menos atención a sus estudios". "Por la falta de atención hacia el docente, no dan buen rendimiento académico"

$32 \%$ de los padres de familia consideró que los niños con TDAH presentan aislamiento. El $21 \%$ consideró que no es una característica del trastorno y el $47 \%$ que no sabe. Lo cual se sustenta en respuestas como: "Muchas veces son cansones, por eso los demás amigos no quieren estar con ellos." "Los humillan, los excluyen."

$42 \%$ de los padres consideraban que los escolares con TDAH tenían dificultades a nivel familiar, mientras que el 58\% consideraba que no sabía si esta consecuencia se presentaba o no, lo cual se expresaba como: "No tenemos la tolerancia para entenderlos." Con relación a los trastornos comórbidos, $5 \%$ de los padres de familia consideraron que los niños con TDAH sí tienen trastornos asociados, $42 \%$ consideró que no y el $53 \%$ que no sabía. $42 \%$ de los padres de familia consideró que los niños con TDAH no tienen consecuencias a largo plazo en la vida, el $32 \%$ consideró que hay consecuencias y el $26 \%$ que no sabe.

Después de las intervenciones educativas, el $92 \%$ de los padres de familia manifestó que su conocimiento sobre el TDAH aumentó mientras que el $8 \%$ dijo que no. Lo cual expresaron como: "No tenía conocimiento del tema"; "No tenía claridad sobre el TDAH"; "Ya sé que es una enfermedad". $83 \%$ de los padres de familia consideró que tiene herramientas para el manejo en casa de un niño con TDAH, mientras que el $17 \%$ considera que no las adquirió. Lo cual se corrobora con respuestas como: "Se puede hacer un mejor trabajo con el niño, acompañado por los profesores y acompañando a otros" "En esta charla, que es la primera para mí, la información hizo detectar la situación de mi hijo".

83\% de los padres de familia consideró que sabe adónde puede llevar a un niño con TDAH, mientras que el $17 \%$ no sabía. Mencionaron diferentes instituciones o profesionales que intervienen en el tratamiento de los niños: identificando especialmente un $39 \%$ al psicólogo como el profesional que interviene en estos casos. $26 \%$ al psiquiatra infantil, $18 \%$ al neuropsicólogo y otros profesionales con un 17\%. Finalmente, 67\% de los padres de familia identificó características del TDAH en sus hijos, mientras que el 33\% no las identificó.

\section{DISCUSIÓN}

Los hallazgos antes de la intervención muestran el desconocimiento de los padres respecto al TDAH, su frecuencia, sus manifestaciones clínicas y las repercusiones académicas y psicosociales; lo cual determina un impacto negativo muy importante tanto para el bienestar 
del menor, como el de su familia. De acuerdo con Cornejo, un niño con TDAH que no recibe un diagnóstico y/o un tratamiento oportuno tiene altas probabilidades de sufrir a lo largo de su desarrollo un notable deterioro en su rendimiento escolar, sus relaciones familiares y su entorno social.(2) Y lo que es más grave, que dicho deterioro y sus consecuencias se extiendan durante su vida adulta en forma de problemas laborales, dificultades de pareja, consumo de drogas, conductas antisociales y otros trastornos psiquiátricos asociados ${ }^{(2)}$. Lo cual evidenció la necesidad de las intervenciones en esta comunidad educativa.

A partir de los resultados obtenidos después de la intervención educativa, los padres demostraron un aumento de bases teóricas y herramientas prácticas con respecto a los conocimientos previos; con lo cual puede afirmarse que la evaluación de las actividades educativas programadas, cumplió los objetivos propuestos. Los padres describieron las características de los escolares con Trastorno de Déficit de Atención con Hiperactividad (TDAH) y su comorbilidad con otros trastornos, haciendo la diferenciación entre ellos. Adquirieron herramientas para el manejo en casa y se enfatizó en la importancia de la remisión temprana para el inicio oportuno del tratamiento multidisciplinar.

La intervención educativa desde enfermería posibilitó el conocimiento del trastorno por parte de los padres, su manejo y remisión. La ganancia de conocimientos aporta mayor información y posibilita un diagnóstico y tratamiento oportuno, lo cual conlleva a la disminución del deterioro tanto escolar como psicosocial en los menores, disminuyendo la posibilidad de alteraciones en la salud mental de este grupo poblacional.

\section{CONCLUSIONES.}

EI TDAH constituye un importante problema de salud en la etapa escolar, el cual debe ser analizado desde tres hechos fundamentales: la alta prevalencia del trastorno y el desconocimiento de los padres sobre él, lo cual conlleva a la falta de remisión de los escolares para su tratamiento o intervención.

La enfermería tiene como una de las premisas básicas la educación de la salud y las estrategias educativas se constituyen en una valiosa herramienta, ya que como profesionales nos concierne implementar actividades dirigidas a los Padres que les permitan conocer las problemáticas actuales de los escolares y por ende su manejo específico en el hogar.

Así mismo, se recomienda realizar actividades educativas orientadas a la formación de los educadores, partiendo de la necesidad de realizar un trabajo conjunto con los padres desde el contexto escolar buscando acciones encaminadas a trabajar en la prevención de las complicaciones del TDAH. Se hace necesaria la creación de canales de comunicación apropiados entre los padres y la institución escolar de tal forma que permita que estos estén en constante comunicación y que se dé la continuidad en la institución de los procesos que se inician en el hogar.

Es importante continuar con estudios en el tema utilizando el corte cualitativo de investigación que permita profundizar en las experiencias de los padres de familias con niños diagnosticados con TDAH respecto a su padecimiento y cuidado, considerando que el tema es de gran importancia para profesionales de salud, educadores y padres. 


\section{REFERENCIAS BIBLIOGRÁFÍCAS.}

1. Bará-Jimenez S, Vicuña P, Pineda D.A, Henao G.C. Perfiles neuropsicológicos y conductuales de niños con trastorno por déficit de atención/hiperactividad de Cali, Colombia. REV NEUROL 2003; 37(7):608-615.

2. Cornejo J.W, Osío O, Sánchez Y, Carrizosa J, et al Prevalencia del trastorno por déficit de atención-hiperactividad en niños y adolescentes colombianos. REV NEUROL 2005; 40 (12): 716-722.

3. Vélez A, Talero C, González R, Ibáñez M. Prevalencia del trastorno por déficit de atención con hiperactividad en estudiantes de escuelas de Bogotá, Colombia. Acta Neurol Colomb 2008; 24 (1): 6-12.

4 Castellanos F.X, Acosta M.T, Neuroanatomía del trastorno por déficit de atención con hiperactividad. REV NEUROL 2004; 38 (Supl 1), S131-S136.

5. Peña J.A, Montiel-Nava C. Trastorno por déficit de atención/hiperactividad: ¿mito o realidad. REV NEUROL. 2003; 36(2): 173-179

6 Artigas-Pallarés J. Comorbilidad en el trastorno por déficit de atención/hiperactividad. REV NEUROL. 2003 ;36 (Supl 1):S68-S78.

7. Sell-Salazar F. Síndrome de Hiperactividad y déficit de atención REV NEUROL. 2003; 37(4):353-358.

8. Rosello B, García-Castellar R, Tárraga-Mínguez R, Mulas F. El papel de los padres en el desarrollo y aprendizaje de los niños con trastorno por déficit de atención con hiperactividad. REV NEUROL 2003; 36 (Supl1):S79-S84.

9. Mena B, Nicolau R, Salat L, Tort P, et al. Guía Práctica para Educadores: El alumno con TDAH. Trastorno por déficit de atención con o sin hiperactividad. $2^{\underline{a}}$ edición. Barcelona: Ediciones Mayo; 2006.

10. SPSS for windows 13.0 Chicago SPSS INC., 2005.

11. Resolución 008430 de 1993, por la cual se establecen las normas científicas, técnicas y administrativas para la investigación en salud. Santa Fe de Bogotá: Ministerio de Salud; 1993.

ISSN 1695-6141

๑) COPYRIGHT Servicio de Publicaciones - Universidad de Murcia 